\title{
The weak density of the non-invertible elements of a commutative Banach algebra
}

\author{
Colin C. Graham
}

\begin{abstract}
This is proved: if $B$ is a commutative Banach algebra with identity, then the non-invertible elements of $B$ are weakly dense in $B$ if and only if the maximal ideal space of $B$ is infinite.
\end{abstract}

THEOREM. Let $B$ be a commutative Banach algebra with identity. Then the non-invertible elements of $B$ are weakly dense in $B$ if and only if the maximal ideal space of $B$ is infinite.

Gray [2] has proved this for the case $B=C(X)$, where $X$ is a compact infinite Hausdorff space. Our proof of the generalized result appears to be simpler than Gray's. Before beginning with two easy lemmas, it is perhaps worth noting that the invertible elements of $C(X)$ are weakly dense (that is, dense in the topology of pointwise bounded sequential convergence) and that the invertible elements are not weakly dense in the "disc algebra" $A$, the algebra of functions continuous on the set of complex numbers of modulus less than or equal to one and analytic on the set of complex numbers of modulus less than one. (The first statement is immediate; the second follows from an application of normal families to any sequence $f$ converging weakly to the function $z$.) See also the discussion of the norm-closure of non-invertible elements in [4, A.1.1], and the beginning of [2].

LEMMA 1. Let $B$ be any Banach algebra with identity 1 , and let Received 7 October 1970. 
$f \in B$ be invertible. Then a net $\left\{f_{\alpha}\right\} \subseteq B$ converges weakly to $f$ if and only if $\left\{f^{-1} f_{\alpha}\right\}$ converges weakly to 1 .

LEMMA 2. Let $B$ be any commutative Banach algebra. Then the following are equivalent:

(i) the maximal ideal space of $B$ is infinite;

(ii) $B$ contains an element with an infinite spectrum;

(iii) the Gel'fand representation of $B$ is infinite dimensional.

We now prove the Theorem. One direction is easy: if the maximal ideal space of $B$ is a finite set $\left\{x_{1}, \ldots, x_{n}\right\}$, let $m_{j}$ $(j=1, \ldots, n)$ be the element of $B^{*}$ corresponding to a unit mass at $x_{j}$. Then we see that if $f \in B$ is non-invertible, then

$$
\sup _{j}\left|\left\langle f, m_{j}\right\rangle-\left\langle 1, m_{j}\right\rangle\right|=1 \text {. }
$$

Hence, the non-invertible elements of $B$ are not weakly dense in $B$.

We now prove the other direction of the Theorem: if the maximal ideal space of $B$ is infinite, then the non-invertible elements of $B$ are weakly dense in $B$. Using Lemma 1 , we see that it will be enough to show that if $m_{1}, \ldots, m_{n} \in B^{*}$ and $\varepsilon>0$, then there exists a non-invertible element $f \in B$ such that

$$
\sup _{j}\left|\left(f, m_{j}\right\rangle-\left\langle 1, m_{j}\right\rangle\right|<\varepsilon \text {. }
$$

Let $R$ be the radical of $B$, and let $m_{j}^{\prime}$ denote the restriction of $m_{j}$ to $R$. We shall s.ay $m_{j}$ is special if $m_{j}^{\prime} \neq 0$. Let $m_{1}, \ldots, m_{k}$ be the special $m_{j}^{\prime}$ s (if any), and assume that $m_{l}^{\prime}, \ldots, m_{l}^{\prime}, l \leq k$ is a maximal linearly independent subset of $\left\{m_{1}^{\prime}, \ldots, m_{k}^{\prime}\right\} \subseteq R^{*}$. Then

$$
m_{j}^{\prime}=\sum_{i=1}^{Z} c_{i j} m_{i}^{\prime}, \quad Z<j \leq k
$$

We define $m_{n+j}=m_{\imath+j}-\sum_{i=1}^{l} c_{i, l+j} m_{i}, 1 \leq j \leq k-\imath$. Then $m_{n+j}$ is not 
special, $1 \leq j \leq k-2$, that is $\left.m_{n+j}\right|_{R} \equiv 0$.

Now choose $\delta>0$ so small that $f \in B$ and

$$
\sup \left\{\left|\left\langle f, m_{j}\right\rangle-\left\langle 1, m_{j}\right\rangle\right|: 1 \leq j \leq \imath, k<j \leq n+k-\imath\right\}<\delta
$$

implies (1).

We now find $f \in B$ satisfying (2), that is we may assume that $m_{1}, \ldots, m_{k}$ are special and $m_{1}^{\prime}, \ldots, m_{k}^{\prime}$ are linearly independent in $R^{*}$, and $m_{k+1}, \ldots, m_{n}$ annihilate $R$.

Because $m_{k+1}, \ldots, m_{n}$ annihilate $R$, they may be considered as elements of $(B / R)^{*}$. Now, $m_{k+1}, \ldots, m_{n}$ generate a finite dimensional closed [3] subspace $S$ of $(B / R)^{*}$. Set ${ }_{S} \perp=\{b \in B / R:\langle b, s)=0, s \in S\}$. Then $(B / R) / S^{\perp}=S^{*}$, so $S^{\perp}$ has finite co-dimension in $B / R$. By Lemma $2, B / R$ is infinite dimensional: there exists $g \neq 0, g \in S^{\perp}$. Because $g \neq 0, g \in B / R$, the Gel'fand transform $\hat{g}$ of $g$ is not identically zero on the Shilov boundary of $B / R$. Choose a point $x$ in the Shilov boundary of $B / R$ and a representative $g_{1}+R$ of $g$ so that $a=\hat{g}_{1}(x) \neq 0$. Set

$$
f_{1}=1-\frac{1}{a} g_{1} \text {. }
$$

Then $\hat{f}_{l}(x)=0$, and $k<j \leq n$ implies

$$
\begin{aligned}
\left\langle f_{1}, m_{j}\right\rangle & =\left\langle 1, m_{j}\right\rangle-\frac{1}{a}\left\langle g_{1}, m_{j}\right\rangle \\
& =\left\langle 1, m_{j}\right\rangle-\frac{1}{a}\left\langle g, m_{j}\right\rangle=\left\langle 1, m_{j}\right\rangle .
\end{aligned}
$$

Thus, if the radical of $B$ were zero, we could find a non-invertible $f \in B$ satisfying (2) for $\delta=0$.

We now set

$$
\alpha_{j}=\left\langle f_{1}, m_{j}\right\rangle, j=1, \ldots, l .
$$

Because $\left\{m_{1}^{\prime}, \ldots, m_{l}^{\prime}\right\}$ is a finite linearly independent set in $R^{*}$, there exists (by Hahn-Banach) an element $v \in R^{* *}$ such that $\left\langle\nu, m_{j}^{\prime}\right\rangle=\alpha_{j}$ $(j=1, \ldots, 2)$. Because $R$ is weak-* dense in $R^{* *}$ [3] there exists 
$r \in R$ such that

$$
\left|\left\langle r, m_{j}^{\prime}\right\rangle-\left\langle v, m_{j}^{\prime}\right\rangle\right|<\delta \quad(j=1, \ldots, Z) .
$$

Set $f=f_{1}-r$. It is now clear from (3), (4) and (5) that (2) holds. Furthermore, $r$ has only zero in its spectrum so the spectrum of $f$ is the spectrum of $f_{1}$, that is $f_{1}$ is non-invertible.

\section{References}

[1] Theodore W. Gamelin, Uniform algebras (Prentice-Hall, Englewood Cliffs, New Jersey, 1969).

[2] J.D. Gray, "The weak closure of the set of singular elements in a Banach algebra", Bull. Austral. Math. Soc. 2 (1970), 89-93.

[3] J.L. Kelley, Isaac Namioka and W.F. Donoghue, Jr, Kenneth R. Lucas, B.J. Pettis, Ebbe Thue Poulsen, G. Baley Price, Wendy Robertson, W.R. Scott, Kennan T. Smith, Linear topological spaces (Van Nostrand, Princeton, New Jersey; Toronto; New York; London; 1963).

[4] Charles E. Rickart, General theory of Banach algebras (Van Nostrand, Princeton, New Jersey; Toronto; London; New York; 1960).

Northwestern University,

Evanston, Illinois,

USA. 